\title{
Thoracic hemivertebra excision in adults via a posterior- only approach
}

\author{
Report of two cases
}

\author{
David W. Polly, JR., M.D., Michael K. Rosner, M.D., William Monacci, M.D., \\ AND Ross R. MOQUIN, M.D.
}

\author{
National Capital Consortium, Neurosurgery Program, Walter Reed Army Medical Center, \\ Washington, $D C$
}

\begin{abstract}
Hemivertebrae are a common cause of congenital scoliosis. Depending on their location and the magnitude of the resultant deformity, they may be asymptomatic or require treatment. In the past, treatment has focused on prevention of deformity progression in growing children. Little has been written about congenital scoliosis presenting in adulthood. Because the aging of the spine is a kyphosing process and hemivertebrae often present with a local segmental kyphotic alignment, this can become symptomatic. Excision of hemivertebrae is well established as a safe and effective procedure when treatment is required. Initially this was conducted via a combined anterior-posterior approach. Recently some authors have indicated that in the lumbar spine hemivertebra resection can safely and effectively be achieved via a single posterior transpedicular approach. The authors report two adult cases in which they performed posterior transpedicular lateral extracavitary excision of a thoracic, fully segmented hemivertebrae. Essentially complete correction of the deformity was achieved. There were no neurological complications. The patients were spared a thoracotomy and no chest tubes were required.
\end{abstract}

KEY WORDS • hemivertebra • spinal deformity • spinal instrumentation

The location and type of vertebral anomaly can affect the severity and prognosis of congenital scoliosis. ${ }^{8-10,13}$ When a large deformity is present at an early age, surgical treatment is necessary. Surgical correction of congenital curves is generally more successful when high-risk curves of a smaller magnitude are treated early., ${ }^{3,4}$ In cases in which the curves manifest later in life, treatment is more challenging to the clinician.

There are a number of surgery-related options available for the management of congenital scoliosis. Historically these options have included posterior spinal fusion with or without instrumentation, anterior and posterior fusion in situ, hemiepiphysiodesis, and fusion following hemivertebra excision via combined anterior-posterior approach. ${ }^{1-7}$ In single-stage hemivertebra excision deformity correction is advantageously combined with a short-segment fusion.

Hemivertebra excision was first reported in the early 20th century ${ }^{12}$. The potential for neurological injury led Leatherman and Dickson ${ }^{6}$ to recommend a staged sequential anterior-posterior hemivertebra resection. In subsequent reports by Bradford and Boachie-Adjei ${ }^{2}$ and others ${ }^{3,5,7}$ the authors reported the safety and efficacy of single-stage combined anterior-posterior excision. These series inclu- ded children who ranged in age from 1 to greater than 10 years. Callahan and coworkers ${ }^{3}$ suggested that results might be improved if surgery was performed at a younger age. The purpose of this report is to present our experience with a posterior-only transpedicular lateral extracavitary hemivertebra excision in two symptomatic adults.

\section{CASE REPORTS}

\section{Treatment Overview}

We report two cases of adults presenting de novo with fully segmented thoracic hemivertebrae in whom symptoms merited surgical treatment. Both patients underwent hemivertebra excision via a posterior-only, transpedicular lateral extracavitary approach at a single institution; the procedures were performed by a single surgical team. The spine was stabilized using pedicle screw fixation above and below the level of resection.

\section{Surgical Technique}

Both hemivertebrae were in the midthoracic spine. Fluoroscopy was used to identify the level of each hemivertebra. The posterior hemilamina was excised. The 
corresponding medial $8 \mathrm{~cm}$ of rib was resected via a lateral extracavitary approach. The suprajacent and subjacent discs were resected. The hemivertebra body was then decancellated and removed piecemeal. Compression on the ipsilateral side was then applied to the pedicle screws above and below the site of resection. If correction was not achieved using minimal force application, additional resection was undertaken until correction was obtained. This required excision of the posterior concave facet joint, which often is ankylosed. Bone graft material derived from the resected body and rib was used to facilitate anterior and posterior fusion. In both cases, motor evoked potentials and somatosensory evoked potentials were used to ensure no neurological compromise occurred.

\section{Case Summaries}

Case 1. This 42-year-old woman suffered constant midthoracic back pain. Pain was worsened by activities such as standing and sitting for prolonged periods of time. Symptoms were alleviated minimally by physical therapy and oral analgesic medication. Imaging revealed a T-6 hemivertebra with resulting kyphoscoliosis (Fig. 1). She was taken to the operating room where she underwent lateral extracavitary excision of the hemivertebra and placement of posterior instrumentation. She required a delayed placement of chest tube for pleural effusion. Serial radiographs revealed good fusion and improvement in kyphoscoliosis (Fig. 2). Although she continued to suffer back pain, her preoperative symptoms nonetheless improved.

Case 2. This 24-year-old woman suffered constant midthoracic back pain and myelopathy. Pain was made worse with activity. She was unable to perform her military duties. Conservative therapy proved ineffective. Neuroimaging revealed a T-5 hemivertebra with resulting kyphoscoliosis (Fig. 3). She underwent lateral extracavitary excision of the hemivertebra and placement of posterior instrumentation. Her postoperative course was complicated by acute cholecystitis unrelated to the surgical procedure. Serial radiographs revealed good fusion and improvement in kyphoscoliosis (Fig. 4). She remains on active duty 30 months after surgery meeting the physical requirements for duty.

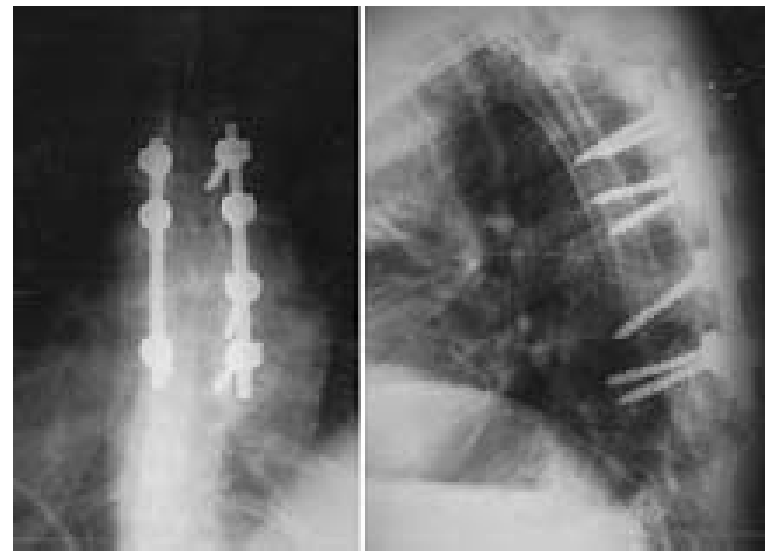

Fig. 2. Case 1. Postoperative radiographs (anteroposterior [left] and [right]).

\section{DISCUSSION}

Fully segmented hemivertebrae can have significant unbalanced growth potential. ${ }^{8-10,13}$ Patients presenting with a high-magnitude curve at a very young age are at substantial risk for deformity progression. Although the optimal treatment for "high-risk" congenital curves is surgery, the required approach remains controversial. Treatment options include posterior spinal fusion alone, anterior-posterior fusion, hemiepiphysiodeses, or singleor two-stage hemivertebra excision and fusion.

Posterior spinal fusion with or without anterior spinal fusion will arrest progression of the curve and may, with the use of instrumentation, obtain curve correction. ${ }^{4}$ Using this method, Winter and Moe ${ }^{14}$ together and with Lonstein $^{15}$ reported their extensive experience in young children and adolescents. They found that in some children undergoing posterior spinal fusion alone "bending of the fusion mass" occurred (or what is now called crankshaft phenomenon) in up to $14 \%$ of cases. Bending of the fusion mass was associated with some loss of spinal alignment correction. In both studies, however, they did not correlate the eventual bending of the fusion mass with posteriorly

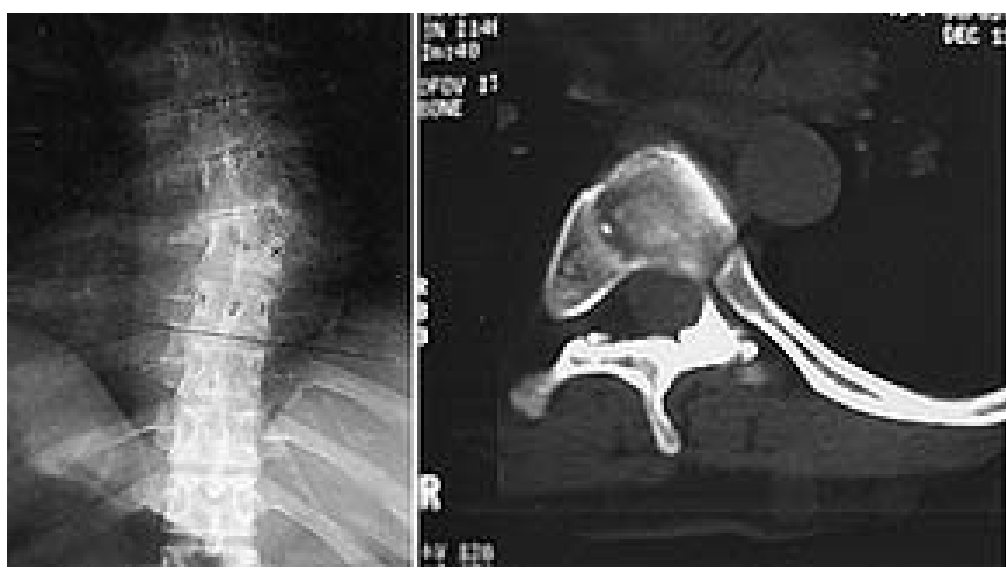

Fig. 1. Case 1. Preoperative studies. Left: Anteroposterior radiograph. phy scans. 

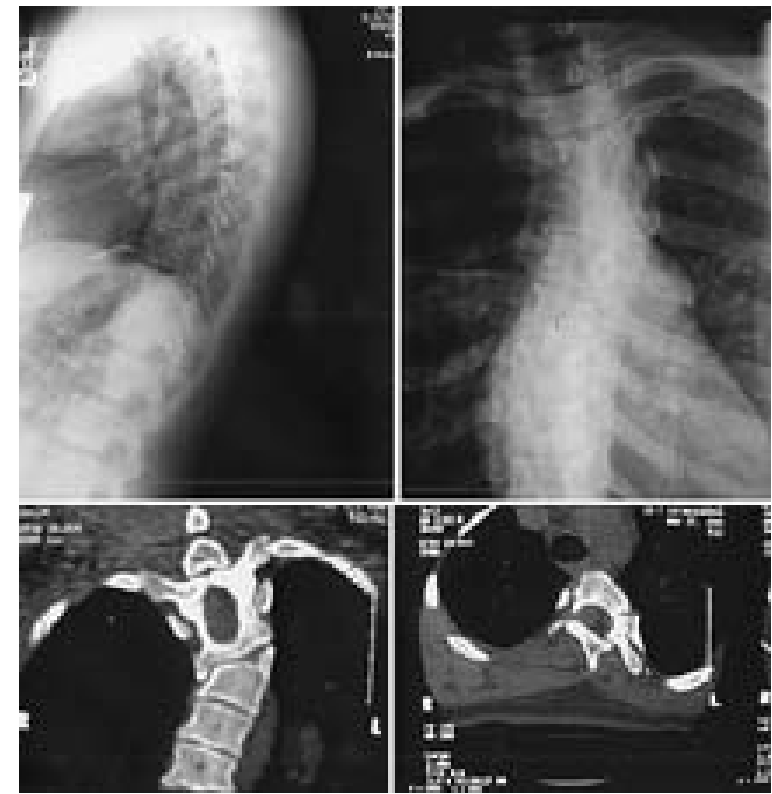

Fig. 3. Case 2. Preoperative radiographs (upper left) and (right) and preoperative computerized tomography scans (lower left) and (right).

tethered anterior growth. They believed that an associated anterior fusion was not necessary. In a similar population, Hall and coworkers ${ }^{4}$ reported that with the use of Harrington instrumentation a greater degree of correction was maintained.

Hemiepiphysiodeses was originally described in $1963 .{ }^{12}$ Bradford $^{1}$ reported on hemiepiphysiodeses in 1982. This procedure was an attempt to arrest curve progression through partial convex anterior hemiarthrosis while achieving a slow curvature correction with further spinal growth. Hemiepiphysiodeses was also an attempt to limit the restriction of growth that occurs after posterior spinal fusion. When used in congenital scoliosis, results of this procedure were mixed.

In the past hemivertebra excision was considered to be an aggressive treatment. Leatherman and Dickson ${ }^{6}$ expressed concern about the neurological risk associated with the single-stage hemivertebra excision. Despite their concern, they suggested that two-stage hemivertebra excision might pose less danger of neurological injury than instrumentation-augmented posterior spinal fusion because hemivertebra excision shortens the spine while correcting the deformity. ${ }^{6}$ Subsequently Bradford and Boachie-Adje ${ }^{2}$ reported that this procedure could be conducted safely in a single stage. The results of a number of other studies of heterogeneous groups of children and adolescents have supported their results. ${ }^{3,4,7,13}$ It is unclear whether this decrease in complications is due to an improvement in surgical or anesthetic technique or neurological monitoring. It is likely that the combination of these advances has contributed to a safer procedure than that originally reported by Leatherman and Dickson.

A one-stage hemivertebra resection via the posterior approach has been previously described..$^{11,12}$ Ruf and Harms ${ }^{11}$ reported on 21 cases (age range $15-167$ months) with a 2-year follow-up period. In all cases a posterior-

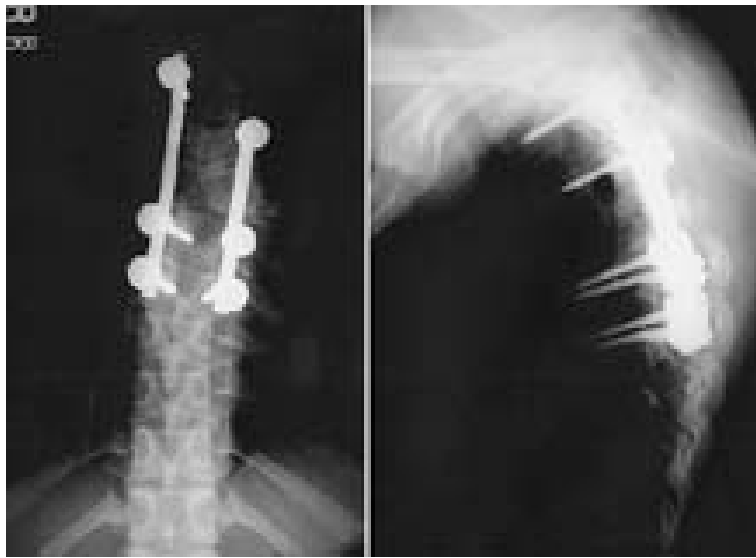

Fig. 4. Case 2. Postoperative radiographs.

only approach was used for the placement of transpedicular instrumentation and hemivertebra resection. The mean preoperative Cobb angle of the main curve was $41^{\circ}$; postoperatively it was $14^{\circ}$ and at the latest follow-up assessment it was $15^{\circ}$. The angle of kyphosis was $24^{\circ}$ before surgery but improved to $11^{\circ}$ after surgery. There was one infection, one pedicle fracture, and two failures of the initially used wire instrumentation. Shono, et al., ${ }^{12}$ reported on 12 patients (age range 8-24 years) in whom hemivertebra resection was performed via a one-stage posterior approach; the minimum follow-up period was 2 years. All patients harbored a single nonincarcerated hemivertebra at T-9 (one patient), T-10 (two patients), T-11 (two patients), T-12 (four patients), and L-1 (three patients)]. ${ }^{12}$ The mean preoperative scoliosis was $49^{\circ}$ and was corrected to $18^{\circ}$, and preoperative kyphosis of $40^{\circ}$ was corrected to $17^{\circ}$. A trunk shift of $23 \mathrm{~mm}$ was improved to $3 \mathrm{~mm}$. No intraoperative complications were noted, and a solid fusion was achieved in all patients.

To date there has been very little written on congenital spinal deformity presenting in adulthood. In the two previous reports the authors concentrated on the adolescent population ${ }^{11,12}$ and little was noted on single-stage resection in the adult population. In our experience, with patients presenting in this fashion there are significant sagittal-plane issues. In both of these patients, there was an acute kyphotic angulation at the site of the hemivertebra. With the aging process the spine becomes progressively more kyphotic, primarily through loss of disc height. Because the discs can no longer compensate for the structural osseous anomalies, degenerative spurring can occur. When this happens in the thoracic spine, cord compression or thoracic nerve root compression can occur. Treatment then is directed at decompressing the neural elements and achieving appropriate spinal realignment while trying to minimize surgery-related morbidity. In our two cases, correcting the scoliosis is helpful, but restoring appropriate segmental sagittal alignment is paramount.

\section{CONCLUSIONS}

Fully segmented thoracic hemivertebrae that require surgical treatment can be resected via a posterior-only approach in not only pediatric patients but also adult pa- 
tients. The combined use of transpedicular decancellation and a lateral extracavitary approach makes the resection safe at the spinal cord level. Rigid internal fixation involving placement of thoracic pedicle screws makes stabilization and rapid postoperative mobilization possible.

\section{Disclosure}

The opinions or assertions contained herein are the private views of the authors and are not to be construed as official or as reflecting the views of the US Army, US Navy, or the Department of Defense. The authors are employees of the US government. This work was prepared as part of their official duties and as such, there is no copyright to be transferred.

\section{References}

1. Bradford DS: Partial epiphyseal arrest and supplemental fixation for progressive correction of congenital spinal deformity. J Bone Joint Surg Am 64:610-614, 1982

2. Bradford DS, Boachie-Adjei O: One-stage anterior and posterior hemivertebral resection and arthrodesis for congenital scoliosis. J Bone Joint Surg Am 72:536-540, 1990

3. Callahan BC, Georgopoulos G, Eilert RE: Hemivertebral excision for congenital scoliosis. J Pediatr Orthop 17:96-99, 1997

4. Hall JE, Herndon WA, Levine CR: Surgical treatment of congenital scoliosis with or without Harrington instrumentation. J Bone Joint Surg Am 63:608-619, 1981

5. Holte DC, Winter RB, Lonstein JE, et al: Excision of hemivertebrae and wedge resection in the treatment of congenital scoliosis. J Bone Joint Surg Am 77:159-171, 1995

6. Leatherman KD, Dickson RA: Two-stage corrective surgery for congenital deformities of the spine. J Bone Joint Surg Br 61:324-328, 1979
7. Leong JC, Day GA, Luk KD, et al: Nine-year mean follow up of one-stage anteroposterior excision of hemivertebrae in the lumbosacral spine. Spine 18:2069-2074, 1993

8. McMaster MJ, David CV: Hemivertebra as a cause of scoliosis. A study of 104 patients. J Bone Joint Surg Br 68:588-595, 1986

9. McMaster MJ, Ohtsuka K: The natural history of congenital scoliosis. A study of two hundred and fifty-one patients. J Bone Joint Surg Am 64:1128-1147, 1982

10. Nasca RJ, Stilling FH III, Stell HH: Progression of congenital scoliosis due to hemivertebrae and hemivertebrae with bars. J Bone Joint Surg Am 57:456-466, 1975

11. Ruf M, Harms J: Hemivertebra resection by a posterior approach: innovative operative technique and first results. Spine 27:1116-1123, 2002

12. Shono Y, Abumi K, Kaneda K: One-stage posterior hemivertebra resection and correction using segmental posterior instrumentation. Spine 26:752-757, 2001

13. Slabaugh PB, Winter RB, Lonstein JE, et al: Lumbosacral hemivertebrae. A review of twenty-four patients, with excision in eight. Spine 5:234-244, 1980

14. Winter RB, Moe JH: The results of spinal arthrodesis for congenital spinal deformity in patients younger than five years old. J Bone Joint Surg Am 64:419-432, 1982

15. Winter RB, Moe JH, Lonstein JE: Posterior spinal arthrodesis for congenital scoliosis. An analysis of the cases of two hundred and ninety patients, five to nineteen years old. J Bone Joint Surg Am 66:1188-1197, 1984

Manuscript received December 16, 2002.

Accepted in final form January 10, 2003.

Address reprint requests to: Ross R. Moquin, M.D., Walter Reed Army Medical Center, 6900 Georgia Avenue NW, Neurosurgery Service, Building 2, Room 6444, Washington, DC, 20307. email: RossMoquin@aol.com. 